DOI: $\underline{\text { https://doi.org/10.24297/jam.v16i0.8217 }}$

\title{
The Primitive and Imprimitive Soluble Subgroups of GL(4,Pk)
}

\author{
Behnam Razzaghmaneshi \\ Assistant Professor of Islamic Azad University, Talesh Branch, Talesh, Iran \\ B_RAZZAGH @ Yahoo.com
}

\begin{abstract}
:
In this paper we will determined all of the primitive and imprimitive Soluble Subgroups of $G L\left(4, p^{k}\right)$. It turns out that the number of types of the irreducible Soluble Subgroups in $\mathrm{GL}\left(4, \mathrm{p}^{\mathrm{k}}\right)$ are 10 types and are $\mathrm{M}_{\mathrm{i}, \mathrm{i}}=1, \ldots, 10$.

moreover we find these subgroups.
\end{abstract}

AMS subject classification Number: 20B32, 20D10.

Keywords and Phrases: Symplectic Groups, General Linear Groups, Primitive Groups.

\section{Section 1: Introduction and Elementary Definitions.}

in the early 1871 Jordan determined a table containing the number of conjugacy classes of maximal irreducible soluble subgroups of $G L(n, p)$, for $p^{n}<106$.([6]) Dickson (1901, Chapter 12, pp. 260-287) determined all subgroups of PSp $\left(2, p^{k}\right)$ and in (1904) he determined all subgroups of PSp $(4,3)$. Mitchell (1914) determined the maximal subgroups of PSp $\left(4, p^{k}\right)$ for odd $p$. Liskovec (1973) classified the maximal Irreducible $(p, q)$ subgroups of $G L\left(r^{2}, p\right)$, where $q$ and or are primes and $q$ is odd. Colon (1977) determined the non - abelian $q$ subgroups (q prime) of $G L\left(q, p^{k}\right)$ and the non - abelion 2 - subgroups of Sp (2, $\left.p^{k}\right)$. Harada and yamaki (1979) determined the irreducible subgroup of $G L(n, 2)$ for $n \grave{A} 6$. KondratĖev $(1985,1986$, and 1987) determined the irreducible subgroups of $G L(7,2)$, the insoluble irreducible subgroups of $G L(8,2)$ and $G L(9,2)$ and the insoluble primitive subgroups of $\mathrm{GL}(10,2)$. In the early 1960 Sims developed an algorithm, based on coset enumeration, which takes as input a group $G$ given by a finite representation and positive integer $n$, and output a list containing a representative of each conjugacy class of subgroups of $G$ whose index is at most $n$. A similar algorithm was developed independently by Schaps (1968). After Kovacs, Neubauer and Newman (unpublished notes) have proposed an algorithm which computing certain maximal subgroups of low index. Now in this paper we will determine the irreducible Soluble Subgroups of $\mathrm{Gl}\left(4, \mathrm{p}^{\mathrm{k}}\right)$. For this purpose, we mention some Definitions and elementary notions.

1.1 Definition: let $G, N$ and $H$ be groups and $G$ has a normal subgroup $N_{0}$ isomorphic to $N$ such that $G / N_{0}$ is isomorphic to $H$, then we write $G=N \lambda H$. If $G$ has a subgroup isomorphic to $H$ which intersects $N_{0}$ trivially then $\mathrm{G}$ is a semidirect product of $\mathrm{N}$ and $\mathrm{H}$, we denote by $\mathrm{G}=\mathrm{N}^{*} \mathrm{H}$.

1.2 Definition : We say that a group $G$ has a central decomposition $\left(H_{1}, \ldots, H_{n}\right)$ if

1 - each $\mathrm{H}_{\mathrm{i}}$ is a normal subgroup of $\mathrm{G}$.

$2-\mathrm{G}=\mathrm{H}_{1} \ldots \mathrm{H}_{\mathrm{n}}$.

3 - for each $i$ and $j, H_{i} \cap H_{j} \leq Z\left(H_{i}\right) \cap Z\left(H_{i}\right)$

4 - for each $i$ and $j, H_{i} \cap H_{j}=Z\left(H_{i}\right)$ or $Z\left(H_{j}\right)$

We also say that $G$ is the central product of $H_{i}$ by $G=H_{1} Y \ldots Y H_{n}$. 
1.3. Definition: The holomorph of group $G$, denote by $H O L(G)$, is the semidirect product of $G$ and its automorphism group.

\section{Section 2 : Notations and Elementary Results.}

In this Section we discuss some necessary results, which needed for later sections to Notations and elementary notions for use of their in after chapter.

2.1. Notation: We use $\operatorname{sym}(X)$ by means the symmetric group on the set $X$, and $S n$ to means that the symetric group on the set of the first $n$ positive integers. If $G$ and $H$ are permutation groups, we denote the wreath product of $\mathrm{G}$ and $\mathrm{H}$ by $\mathrm{G}$ wr $\mathrm{H}$, where $\mathrm{G}$ is a co-ordinate subgroup and $\mathrm{H}$ is the top group.

2.2. Theorem: (Huppert (1967, Theorem II. 3. 2. p. 159). Let $P$ be a prime, $n$ top a positive integer and $V$ be the vector space of dimention $n$ over the field of $p$ elements. If $G$ is a subgroup of $G L(V)$, denote by $V * G$ the permutation group of degree $p^{n}$ which is the semidirect product of $V$ (acting on itself by translation) and $G$ (acting in natural way) considered as a subgroup of $\operatorname{sym}(\mathrm{V})$. Let $S$ be a complete and irredundant set of conjugacy class representatives of the irreducible soluble subgroups of $\mathrm{GL}(\mathrm{V})$.

(a) If $G \in S$. Then $V * G$ is a primitive soluble permutation group of degree $p^{n}$.

(b) If $G \in S$ and $H$ is a subgroup of $G L(V)$, that is conjugate to $G$, then $V * H$ is conjugate in sym(V) to $V * G$.

(c) If $G \in S$ and $H$ is a subgroup of $G I(V)$, that is not conjugate to $G$, then $V * H$ is not conjugate to $V * G$.

(d) If $P$ is a primitive soluble subgroup of $\operatorname{sym}(V)$, then there is a group $G$ in $S$ such that $V$ * $G$ is conjugate to $P$.

We always take $\mathrm{F}$ to be a finite field with $\mathrm{p}^{\mathrm{k}}$ elements and $\mathrm{n}$ a positive integer.

2.3. Theorem (See [4], [5] \& [6]) : There exists an irreducible cyclic subgroup of order $m$ in $G L(n, F)$ if and only if $m$ divides $p^{k n}-1$ and $m$ dos not devide $p^{k d}-1$, for any positive integer $d<n$.

2.4. theorem (See [4], [5] \& [6]): If there exist irreducible cyclic subgroups of order $m$ in $G L(n, F)$ then they lie in a single conjugacy class.

2.5. Definition: $\ln G L(n, F)$ the irreducible cyclic subgroups of order $P^{k n}-1$ are called the signer cycles.

2.6. Definition: An extra special q-group is a finite non abelian q-group whose center, derived group and Frattini subgroup coincide and have order $\mathbf{q}$.

\subsection{Theorem: (See [3])}

(a) Let $G$ be an extraspinal $q$ - group of order $q 1+2 L$ and exponent $q$ or 4 . The group of automorphism $S$ of $G$ which acts trivially on both $Z(G)$ and $G / Z(G)$ is equal to $\operatorname{lnn}(G)$. Let $H$ be the normal subgroup of Aut $(G)$ consisting of those elements that act trivially on $Z(G)$.

Then $\mathrm{H} / \mathrm{Inn}(\mathrm{G})$ is isomorphic to a subgroup of the symplectic group Sp $(2 \mathrm{l}, \mathrm{q})$.

If $q$ is odd, then $H / \operatorname{lnn}(G)$ is isomorphic to the full symplectic group sp (2l, q), If $G$ is the central product of I copies of $D 8$, then $H / \operatorname{lnn}(G)$ is isomorphic to the orthogonal group $\mathrm{O}^{+}(2 \mathrm{l}, 2)$. If $\mathrm{G}$ is the central product of (I.1) copies of $D 8$ and one $Q_{8}$, then $H / \operatorname{lnn}(G)$ is isomorphic to the orthogonal group $\mathrm{O}^{-}(2 \mathrm{l}, \mathrm{Z})$ 
(b) Let $G$ be the central product of a cyclic group of order 4 and extra special 2-group. The group of automorphisms of $G$ that act trivially on both $Z(G)$ and $G Z(G)$ is equal to $\operatorname{lnn}(G)$. If $H$ is the normal subgroup of Aut $(G)$ consisting the those elements that act trivially on $Z(G)$, then $H / \operatorname{lnn}(G)$ is isomorphic to the symplectic group Sp (2l,2).

Note that the group $\mathrm{O}^{+}(2 \mathrm{l}, 2)$ is the group of all linear transformations that preserve the quadratic form: $f\left(x_{1}, \ldots\right.$, $\left.\mathrm{x}_{21}\right)=\mathrm{x}_{1} \mathrm{x}_{2}+\ldots+\mathrm{x}_{21-1} \mathrm{x}_{21}$ and the group $\mathrm{O}^{-}(2 \mid, 2)$ is the group of all linear transformations that preserve the quadratic form:

$f\left(x_{1}, \ldots, x_{21}\right)=x_{1} x_{2}+\ldots+x_{21-1} x_{21}+x^{2} 21-1+x^{2} 21$

2.8. Definition: Let $G$ be an irreducible subgroup of $G L(n, F)$, acting on the vector space $V$. We call $G$ imprimitive if there exists a decomposition $\mathrm{V}=\mathrm{V}_{1}+\ldots+\mathrm{V}_{\mathrm{r}}(\mathrm{r}>1)$ of $\mathrm{V}$ that is preserred under the action of $\mathrm{G}$.

We call the set $\left\{V_{1} \ldots V_{2}\right\}$ a system of imprimitivity for $G$, and each member of this set is called a block of imprimivity for $G$. The minimum of the set of dimensions of the blocks of imprimitivity for $G$ is called the minimal block size of $\mathrm{G}$. If $\mathrm{G}$ is not imprimitive. We call $\mathrm{G}$ primitive.

2.9. Theorem $\mathbf{E}$ :(see suprunenko (1976, theorem 15. 4. P.109) [11])

let $M$ be an Imprimitive Maximal soluble subgroup of $G L(n, F)$, and let $\Gamma:=\left\{V_{1} \ldots V_{2}\right\}$ be an undefinable system of imprimitivity for $\mathrm{M}$. Let $\theta: \mathrm{M} \rightarrow \operatorname{Sym}(\Gamma)$ be the homomorphism defined by: $\forall g \in M, V_{i}(g \theta):=V_{i g}$. Then $\left.N_{M}\left(V_{1}\right)\right|_{V 1}$ is a primitive maximal soluble subgroup of $G L\left(V_{1}\right), M \theta$ is a transitive maximal soluble subgroup of $\operatorname{sym}(\Gamma)$, and $M$ is linearly isomorhic to $\left.N_{M}\left(V_{1}\right)\right|_{v_{1}} \operatorname{Wr} M \theta$.

2.10. Remark: Consider the case when $m=n$ in the above theorem, then $V_{1}$ is 1 -dimensional, and so $N_{M}$ $\left.\left(V_{1}\right)\right|_{V 1}=G L(I, F)$, by hypothesis $M$ is irreducible, and therefore we must have $p^{k}>2$. In particular $G L(n, 2)$ contains no imprimitive subgroups if $\mathrm{n}$ is prime.

2.11. Theorem (see [4]): Let $m$ be a proper divisor of $n$, let $P_{m}$ be a completed and irredundant set of conjugacy class representatives of the primitive maximal soluble subgroups of $G L(m, F)$ and let $\tau_{\frac{n}{m}}$ be a complete and irredundant set of conjugacy class represtatives of the transitive maximal soluble subgroups of $S_{\underline{n}}$. Define the set $\mathrm{S}_{\mathrm{m}}$ of imprimitive soluble subgroups of $\mathrm{GL}(\mathrm{n}, \mathrm{F})$ by $\mathrm{S}_{\mathrm{m}}:=\left\{\mathrm{P}\right.$ wr $\left.T \mid \mathrm{P} \in \mathrm{P}_{\mathrm{m}}, T \in_{\frac{\tau_{\frac{n}{m}}}{m}}\right\}$.

However, if $p^{k}=2$, then define the set $S_{1}$ to be empty. Let $S$ be the union of the $S m$ as $m$ runs through the proper divisors of $n$. Then those members of $S$ that are maximal soluble from a complete and irredundant set of conjugacy class representatives of the imprimitive maximal soluble subgroups of $G I(n, F)$.

2.12. Theorem: (See [13], lemma 19. 1. P.129, Theorem 20. 9, P.145). Let $A$ be a maximal abelian normal subgroup of $\mathrm{M}$. Then the following statements hold:

(a) A is conjugate to a group of block diagonal matrices, where each blak is the same, and is $\mathrm{m}$ by $\mathrm{m}$, where $\mathrm{m}$ is a divisor of $n$ :

(b) The linear span $\mathrm{E}$, of the powers of any one of the $\mathrm{m}$ by $\mathrm{m}$ diagonal blocks of $\mathrm{A}$ is an extension field of $\mathrm{Fl}_{\mathrm{m}}$.

(c) The degree of this field extension is $\mathrm{m}$. 
(d) $\mathrm{A}$ is isomorphic to the multiplicative group of $\mathrm{E}$ : in particular, $\mathrm{A}$ is cyclic of order $\mathrm{p}^{\mathrm{km}}-1$.

(e) $\mathrm{A}$ is the unique maximal abelian normal subgroup of $\mathrm{M}$.

2.13 : Notation: Define the map $\rho: N_{L}(F) \rightarrow G L(2 l, q)$ by

$$
\rho(g)=\left[\begin{array}{ccccc}
\alpha_{11} & \beta_{11} & \cdots & \alpha_{1 l} & \beta_{1 l} \\
\gamma_{11} & \delta_{11} & \cdots & \gamma_{1 l} & \delta_{1 l} \\
\vdots & \vdots & \vdots & \vdots & \vdots \\
\alpha_{l 1} & \beta_{l 1} & \cdots & \alpha_{l l} & \beta_{l l} \\
\gamma_{l 1} & \delta_{l 1} & \cdots & \gamma_{l l} & \delta_{l l}
\end{array}\right]
$$

$$
u_{i}^{g}=u_{1}^{\alpha_{i 1}} v_{1}^{\beta_{i 1}} \cdots u_{1}^{\alpha_{i l}} v_{l}^{\beta_{i l}} z^{\lambda_{i}}
$$

where: $v_{i}^{g}=u_{1}^{\gamma_{i 1}} v_{1}^{\delta_{i 1}} \cdots u_{1}^{\gamma_{i l}} v_{l}^{\delta_{i l}} z^{\mu_{i}}$

$$
z^{g}=z^{v}
$$

\subsection{Theorem (See to [4], [5] \& [6]):}

Let $n=q^{\prime} m$. Where $1>0$, and $q$ is a prime divisor of $p^{k m}-1$. If $q=2$, then suppos in addition that $P^{k m} \equiv 1(\bmod 4)$. Let $z 1$ be our fixed generator of a singer cycle of $G L(m, F)$, and let $a_{1}$ be our fixed element of order $m$ in $G L(m$, F) such that $a_{1}{ }^{-1} z_{1} a_{1}=z_{1}^{p^{k}}$. Let $a_{1} z$ be the $n$ by $n$ block diagonal matrices with $a_{1}$ and $z_{1}$ running down their diagonals, respectively. Define the matrices $u_{i}$ and $v_{i}$ as Notatin 2.13 . Let $S$ be the subgroup of $G L(2 l, q)$ That is generated by $S p(2 \mathrm{l}, \mathrm{q})$ and the block diagonal matrix with the matrix $\left[\begin{array}{cc}1 & 0 \\ 0 & p^{k}\end{array}\right]$ running down its diagonal. let $\mathrm{D}$ be a completely reducible (not necessarily maximal) soluble subgroup of $S$ which does not fix any non - zero isotropic subspace of the natural module for $S p(2 l, q)$.

Suppose $D$ has generating set $\left\{d_{1}, \ldots, d_{r}\right\}$. If $d_{i}$ is the matrix

$$
\left[\begin{array}{ccccc}
\alpha_{11} & \beta_{11} & \cdots & \alpha_{1 l} & \beta_{1 l} \\
\gamma_{11} & \delta_{11} & \cdots & \gamma_{1 l} & \delta_{1 l} \\
\vdots & \vdots & \vdots & \vdots & \vdots \\
\alpha_{l 1} & \beta_{l 1} & \cdots & \alpha_{l l} & \beta_{l l} \\
\gamma_{l 1} & \delta_{l 1} & \cdots & \gamma_{l l} & \delta_{l l}
\end{array}\right]
$$

Then $g_{i}$ be any matrix of $G L(n, F)$ statistying

$$
\begin{aligned}
& u_{j}^{g_{i}}=u_{1}^{\alpha_{j 1}} v_{1}^{\beta_{j 1}} \cdots u_{l}^{\alpha_{j l}} v_{l}^{\beta_{j l}} z^{\lambda_{j}} \\
& v_{j}^{g_{i}}=u_{1}^{\gamma_{j 1}} v_{1}^{\delta_{j 1}} \cdots u_{l}^{\gamma_{j l}} v_{l}^{\delta_{j l}} z^{\mu_{j}}
\end{aligned}
$$

for some (arbitrary) integer $\gamma_{j}$ and $\mu_{j}$. let $P$ the subgroup of $G L(n, F)$ defined by: $P:=<G_{<a>}\left(V_{1}, \ldots, V_{1}\right), g_{1}, \ldots, g_{r}, u_{1}, v_{1}, \ldots, u_{1}, V_{1}, Z>$ then $P$ is the complete inverse 
image of $\mathrm{D}$ under $\mathrm{r}$. Furthermore, $\mathrm{P}$ is primitive and has a maximal abelian normal

subgroup of order $\mathrm{P}^{\mathrm{km}}$-1. Now let $\mathrm{D}_{1}$ be a complete and irredundant set of $\mathrm{S}$ - conjugacy class representatives of the completely reducible maximal soluble subgroup of $S$ which do not fix any non - zero isotropic subspace of the natural module for $\mathrm{sp}(2 \mathrm{l}, \mathrm{q})$. Let $\mathrm{P} 1$ be the set of groups $\mathrm{P}$ obtained by the above method, one for each $D$, where $D$ runs through the members of $D_{1}$. No two members of $P$ are conjugate in $G L(n, F)$. If $M$ is a primitive maximal soluble subgroup of $G L(n, F)$ whose unique maximal abelian normal subgroup has order $\mathrm{Pkm}_{-} 1$, then $M$ is conjugate to a member of $P_{1}$.

2.15. Theorem: Let $n=2^{1} m$, and suppose that $p^{k m} \equiv 3(\bmod 4)$. Let $z$ be our fixed generator of a singer cycle of $G L(m, F)$, and let $a_{1}$ be our fined element of order $m$ in $G L(m, F)$ such that $a_{1}{ }^{-1} z_{1} a_{1}=z_{1}^{p^{k}}$. Let a and $z$ be the $n$ by $n$ block diagonal matriees with $a_{1}$ and $z_{1}$ running down their diagonals, respectively For $1 \leq i \leq l-1$ define the matrices $u_{i}$ and $v_{i}$ as notation 2.13 .

Define $\mathrm{u}_{1}^{+}$and $\mathrm{v}_{1}^{+}$by: $u_{1}^{+}=\left[\begin{array}{cc}0 & -I_{m} \\ I_{m} & 0\end{array}\right] \otimes I_{2}^{(l-1)}$ and $v_{1}^{+}=\left[\begin{array}{cc}0 & -I_{m} \\ I_{m} & 0\end{array}\right] \otimes I_{2}^{(l-1)}$ and definee. $\mathrm{u}_{1}^{-}$and $\mathrm{v}_{1}^{-}$by $u_{1}{ }^{-}=\left[\begin{array}{cc}0 & -I_{m} \\ I_{m} & 0\end{array}\right] \otimes I_{2}^{(l-1)}$ and $v_{1}^{-}=\left[\begin{array}{cc}\alpha & \beta \\ \beta & \alpha\end{array}\right] \otimes I_{2}{ }^{(l-1)}$ Where $\alpha$ and $\beta$ are two elements of $\mathrm{FI}_{\mathrm{m}}$ such that $\alpha^{2}+\beta^{2}=-I_{m}$. Let $D$ be a completely reducible (not necessarily maximal) soluble subgroup of $\mathrm{O}^{+}(21,2)$ or $\mathrm{O}^{-}(21,2)$ which does not fix any non-zero isotropic subspace of the natural module for the relevant orthogonal group. Suppose $D$ has generating set $\left\{d_{1}, \ldots, d_{r}\right\}$. If $d_{i}$ is the matrix

$$
\left[\begin{array}{ccccc}
\alpha_{11} & \beta_{11} & \cdots & \alpha_{1 l} & \beta_{1 l} \\
\gamma_{11} & \delta_{11} & \cdots & \gamma_{1 l} & \delta_{1 l} \\
\vdots & \vdots & \vdots & \vdots & \vdots \\
\alpha_{l 1} & \beta_{l 1} & \cdots & \alpha_{l l} & \beta_{l l} \\
\gamma_{l 1} & \delta_{l 1} & \cdots & \gamma_{l l} & \delta_{l l}
\end{array}\right]
$$

Then let $g_{i}$ be any matrix of $G L(n, F)$ satisfying

$$
\begin{aligned}
& u_{j}^{g_{i}}=u_{1}^{\alpha_{j 1}} v_{1}^{\beta_{j 1}} \cdots\left(u_{l}^{*}\right)^{\alpha_{j l}}\left(v_{l}{ }^{*}\right)^{\beta_{j l}} z^{\lambda_{j}} \\
& v_{j}^{{ }^{g_{i}}}=u_{1}^{\gamma_{j 1}} v_{1}^{\delta_{j 1}} \cdots\left(u_{l}^{*}\right)^{\gamma_{j l}}\left(v_{l}^{*}\right)^{\delta_{j l}} z^{\mu_{j}}
\end{aligned}
$$

for some (arbitrary) integers $\lambda_{\mathrm{j}}$ and $\mu_{\mathrm{j}}$, and where the superscript * is replaced by + or - according as $D$ belongs to $\mathrm{O}^{+}(21,2)$ or $\mathrm{O}^{-}(21,2)$, respectively, let $\mathrm{P}$ be the subgroup of $\mathrm{GL}(\mathrm{n}, \mathrm{F})$ defined by $\mathrm{P}:=\left\langle\mathrm{C}_{\langle a}\right\rangle \quad\left(\mathrm{V}_{1}, \ldots, \mathrm{V}_{1}^{*}\right)$, $\mathrm{g}_{1}, \ldots, \mathrm{g}_{\mathrm{r}}, \mathrm{u}_{1}, \mathrm{v}_{1}, \ldots, \mathrm{u}_{1}{ }^{*}, \mathrm{v}_{\mathrm{l}}{ }^{*}, \mathrm{z}>$ then $\mathrm{P}$ is the complete inverse image of $\mathrm{D}$ under $\mathrm{r}$. Furthermore, $\mathrm{P}$ is primitive and has a maximal abelian normal subgroup of order $\mathrm{P}^{\mathrm{km}}-1$. now let $\mathrm{D}^{+}$be a complete and irredundant set of $\mathrm{O}^{+}(21,2)$ conjugacy class representatives of the completely reducible maximal soluble subgroups of $\mathrm{O}^{+}(2 \mathrm{l}, 2)$ which do not fix any non-zero isotropic subspace of the natural module for $\mathrm{O}^{+}(2 \mathrm{l}, 2)$.

Define $D^{-}$similarly, with $\mathrm{O}^{-}(21,2)$ in place of $\mathrm{O}^{+}(21,2)$, let $\mathrm{P}_{1}$ be the set of groups $\mathrm{P}$ obtained by the above method, one for each $D$, where $D$ runs through the members of $D^{+}$and $D^{-}$. No two members of $P_{1}$ are conjugate in $G L(n, F)$. If $M$ is a primitive maximal soluble subgroup of $G L(n, F)$ whose unique maximal a belian normal subgroup hos order $P^{\mathrm{km}}-1$. Then $\mathrm{M}$ is conjugate to a member of $\mathrm{P}_{1}$.

Proof: The proof of this theorem goes exactly the similar with the proof previous theorem and the reader can be referred to [4] \& [5]. 
2.16. Definition: Any group constructed by the methods theorems $2.11,2.14$ and 2.15 will be called a JSmaximal (for Jordan-suprunenko) of $\mathrm{GL}(\mathrm{n}, \mathrm{F})$. we will also use the terms JS-imprimitive and JS-primitive to denote imprimitive and primitive JS-maximal, respectively. Note that every JS-maximal is irreducible and soluble. but not necessarily maximal soluble. The smallest value of $\mathrm{p}^{\mathrm{kn}}$ for which there are JS-maximal and are not maximal soluble is 9. (see [11])

2.17. Remark: For the imprimitive we use $P$ wr $T$ where $P$ and $T$ are as described in Theorem $A$ and For the primitives, we use $\left(C_{p^{k m}-1} Y E\right) N D$ where $E$ is extraspinal of order $q^{1+2 l}$ and exponent $q$ or 4 , and $D$ is as described in theorem B or C. Of course, there may be many (pairwise non - isomorphic) groups with than a normal subgroup isomorphic to $C_{p^{k m}-1} \mathrm{YE}$ whose quotient is isomorphic to $\mathrm{D}$. But we always mean the one obtained by the construction methods in this paper.

2.18. Definition: Let the JS - maximal $S$ of $G L(n, F)$ be $M_{1}, \ldots, M_{m}$ and let $G$ be an irreducible soluble subgroup of $G L(n, F)$. If $n$ is prime and $G$ is cyclic, then we define the guardian of $G$ to be that JS - maximal which is the normalizer of a singer cycle. Otherwise the guardian of $G$ is defined to be $M_{i}$, where $i$ is the least positive integer such that $\mathrm{G}$ is $\mathrm{GL}(\mathrm{n}, \mathrm{F})$ - conjugate to a subgroup of $\mathrm{M}$.

\section{Section 3.}

In this Section by using previous theorems and methods of Section $\mathbf{1}$ and section $\mathbf{2}$, We determine The irreducible Soluble Subgroups of $\mathrm{GL}\left(4, \mathrm{p}^{\mathrm{k}}\right)$. For this we proof the following main theorem.

3.1. theorem: Let $p$ be a prime number and $k, n$ be positive integers and let $F$ be the filed of $p^{k}$ elements. Then the number of types of The irreducible Soluble Subgroups in the group $G L\left(4, p^{k}\right)$ is 10 .

Proof: By definition 2.16 since every JS-maximal is irreducible and Soluble, therefore, we suffices to determine the JS-maximal soluble subgroups of the $G L\left(4, p^{k}\right)$. For this purpose, let $F$ be the filed of $p^{k}$ elements. Since both $\mathrm{GL}(1, \mathrm{~F})$ and $\mathrm{S}_{2}$ are Soluble, therefore by theorem (2.11) and remark (2.17) there is exactly one Js-imperative soluble subgroup of $G L(2, F)$, namely, $M_{1}\left(2, p^{k}\right):=G L\left(1, p^{k}\right)$ wr $S_{2}, p^{k} \neq 2$

Also since the unique maximal abelian normal subgroup of the group $G L\left(2, p^{k}\right)$ order $p^{2 k}-1$ or $p^{k}-1$, then by theorems $2.14,2.15$ and remark 2.17 the JS-Primitive group of order $p^{2 k}-1$ and $p^{k}-1$,as follows:

$$
\begin{aligned}
& \mathrm{M}_{2}\left(2, \mathrm{p}^{\mathrm{k}}\right):=C_{p^{2 k-1}}{ }^{*} C_{2,} \\
& \mathrm{M}_{3}\left(2, \mathrm{p}^{\mathrm{k}}\right):=\left(C_{p^{k}-1} \mathrm{Y} \mathrm{Q}_{8}\right) \mathrm{NO}^{-}(2,2), \mathrm{p}^{\mathrm{k}} \equiv 3(\bmod 4) \\
& \mathrm{M}_{4}\left(2, \mathrm{p}^{\mathrm{k}}\right):=\left(C_{p^{k-1}} \mathrm{Y} \mathrm{Q}_{8}\right) \mathrm{N} \mathrm{Sp}(2,2), \mathrm{p}^{\mathrm{k}} \equiv 1(\bmod 4)
\end{aligned}
$$

Therefore by used from JS - maximal $\mathrm{GL}\left(2, \mathrm{p}^{\mathrm{k}}\right)$, the JS-imprimitives of $\mathrm{GL}\left(4, \mathrm{p}^{\mathrm{k}}\right)$ listed as follows.

$$
\begin{aligned}
& M_{1}\left(4, p^{k}\right):=G L\left(1, p^{k}\right) \text { wr } S_{4} \\
& M_{2}\left(4, p^{k}\right):=M_{2}\left(2, p^{k}\right) \text { wr } S_{2}, \\
& M_{3}\left(4, p^{k}\right):=M_{3}\left(2, p^{k}\right) \text { wr } S_{2}, p^{k} \equiv 3(\bmod 4), \\
& M_{4}\left(4, p^{k}\right):=M_{4}\left(2, p^{k}\right) \text { wr } S_{2}, p^{k} \equiv 1(\bmod 4),
\end{aligned}
$$

And also The JS - primitive of $\mathrm{Gl}\left(4, \mathrm{p}^{\mathrm{k}}\right)$ are listed below: 


$$
\begin{aligned}
& \mathrm{M}_{5}\left(4, \mathrm{p}^{\mathrm{k}}\right):=C_{p^{4 k}-1}{ }^{*} \mathrm{C}_{4} \\
& M_{6}\left(4, p^{k}\right):=M_{2}\left(2, p^{k}\right) * C_{2}, p^{k} \neq 2, \\
& \left.\mathrm{M}_{7}\left(4, \mathrm{p}^{\mathrm{k}}\right):=C_{p^{k}-1} \mathrm{Y} \mathrm{D}_{8} \mathrm{Y} \mathrm{Q}_{8}\right) \mathrm{N} \mathrm{O}^{+}(4,2), \mathrm{p}^{\mathrm{k}} \equiv 3(\bmod 4), \\
& \left.\mathrm{M}_{8}\left(4, \mathrm{p}^{\mathrm{k}}\right):=C_{p^{k}-1} \mathrm{Y} \mathrm{D}_{8} \mathrm{Y} \mathrm{Q}_{8}\right) \mathrm{N} \mathrm{HOL}\left(C_{5}\right), \mathrm{p}^{\mathrm{k}} \equiv 3(\bmod 4), \\
& \left.\mathrm{M}_{9}\left(4, \mathrm{p}^{\mathrm{k}}\right):=C_{p^{k}-1} \mathrm{Y} \mathrm{D}_{8} \mathrm{Y} \mathrm{Q}_{8}\right) \mathrm{N} \mathrm{O}^{+}(4,2), \mathrm{p}^{\mathrm{k}} \equiv 1(\bmod 4), \\
& \left.\mathrm{M}_{10}\left(4, \mathrm{p}^{\mathrm{k}}\right):=C_{p^{k}-1} \mathrm{Y} \mathrm{D}_{8} \mathrm{Y} \mathrm{Q}_{8}\right) \mathrm{N} \mathrm{HOL}\left(C_{5}\right), \mathrm{p}^{\mathrm{k}} \equiv 1(\bmod 4) .
\end{aligned}
$$

When $\mathrm{p}^{\mathrm{k}}=2$, then there are exactly 2 - irreducible Soluble Subgroup, namely $\mathrm{M} 2$ and $\mathrm{M}_{5}$.

When $p^{k}=3$, then there are exactly 7 - irreducible Soluble Subgroup, namely $M_{1}, M_{2}, M_{3}, M_{5}, M_{6}, M_{7}$, and $M_{8}$.

When $p^{k}=4$, then there are exactly 4 - irreducible Soluble Subgroup, namely $M_{1}, M_{2}, M_{5}$ and $M_{6}$.

When $p^{k}=5$, then there are exactly 7 - irreducible Soluble Subgroup, namely $M_{1}, M_{2}, M_{4}, M_{5}, M_{6}$.

and $\mathrm{M}_{10}$

When $p^{k}=6$, then there are exactly 4 - irreducible Soluble Subgroup, namely $M_{1}, M_{2}, M_{5}, M_{6}$.

When $p^{k}=7$, then there are exactly 7 - irreducible Soluble Subgroup, namely $M_{1}, M_{2}, M_{3}, M_{5}, M_{6}, M_{7}$ and $M_{8}$.

When $p^{k}=8$, then there are exactly 4 - irreducible Soluble Subgroup, namely $M_{1}, M_{2}, M_{5}, M_{6}$.

When $p^{k}=9$, then there are exactly 7 - irreducible Soluble Subgroup, namely $M_{1}, M_{2}, M_{4}, M_{5}, M_{6}, M_{9}, M_{10}$.

\section{Reference:}

1. J.Dieudonne. "Notes sur les travaux de C. jordan relatifs a la theory des groups finis", in oeuvres de Camille jordan, tome 1, Gauthier - Villars, paris, PP.XVII - XLII(1961).

2. john D.Dixon (1971), the structure of linear groups, Van Nostrand Reinhold, London.

3. john D.Dixon and Brian Mortimer "The primitive permeation groups of degreeless than 1000", Math. proc. comb. phlios. soc. 103, 213-238(1988).

4. B.Huppert" Endliche Gruppen I", springer - Verlag, Berlin, Heidlberg (1967).

5. M. Issacs. "Character degress and derived length of a solvable group" caned. J.Math. 27, 146-151 (1975).

6. C.Jordan,"Sur la resolution des equations les unes par les autres",C.R.Acad.Sci.72,283-290(1871).

7. L.G.Kovacs,J.Neubuser and M.F.Newman,"Some algorithms for finite soluble groups", C.R.Acad.Sci.57,223-232(1828).

8. A.S.KondratÅev, "Irreducible subgroups of the group GL(9, 2)", Mat. Zametki 39,320-329 (1986) 
9. $\quad$ A.S.KondratÅev. "Irreducible subgroups of the group GL(7, 2)". Mat Zameki, 37,317-321 (1985).

10 .A.S.KondratÅev. "The irreducble subgroups of the group GL8(2)"Comm. Aljebra15, 1039-1093 (1987).

11. M.W.Short. "The primitive soluble permutation Groups of degree less than 256,Springer - verlag lecture Notes in Mathematics, 1519 (1992).

12. D.A.Suprunenko (1963), soluble and Nilpotent linear groups, Translations of mathematical monographs, vol. 9, American mathematical society, providence, Rhode Island.

13. D.A.Suprunenko, Matrix groups, Translations of mathematical monographs, vol.45, American mathematical society, providence, Rhode Island (1976).

14. Michio Suzuki, Group theory l, springer verlag, New - York (1981).

15. Michio Suzuki (1986), Group theory II, springer - verlag, New - York.

16. Hans J. Zassenhaus, the theory of Groups, chelsea publishing company, New Youk(1958).

17. B. Razzaghmaneshi, Determination of the JS-maximal soluble subgroups of the general linear group of less than 25 over a field of $\mathrm{p}^{k}$ elements, Ph.D. theses of AUH, university (2002) 